\title{
Aliança Terapêutica nas Terapias Cognitivo-comportamentais por Videoconferência: uma Revisão da Literatura
}

\author{
Bianca Aparecida Ribeiro Singulane \\ Universidade Federal de Juiz de Fora, MG, Brasil.
}

\author{
Laisa Marcorela Andreoli Sartes \\ Universidade Federal de Juiz de Fora, MG, Brasil.
}

\begin{abstract}
Resumo: Nos últimos anos houve um aumento do número de usuários da internet no Brasil e no mundo, enquanto as intervenções por videoconferência surgiram como formas de tratamento para diversos transtornos mentais. Sabe-se que a aliança terapêutica é um componente essencial para se implementar a prática psicoterapêutica, porém, não há um consenso sobre sua formação na psicoterapia por videoconferência. Portanto, o presente artigo teve como objetivo realizar uma revisão de literatura acerca da formação e papel da aliança terapêutica nas Terapias Cognitivo-comportamentais (TCC) por videoconferência. Para isso, foi realizada uma busca em quatro bases de dados, Pubmed, Psycarticles, Lilacs e SciELO, com descritores específicos dos dicionários de cada base. No total, foram encontrados 365 artigos, mas apenas nove preencheram os critérios de inclusão baseados no objetivo deste estudo. Observou-se que a maioria dos artigos indicou que a aliança foi alta no decorrer das sessões de TCC por videoconferência, podendo ser comparada com a aliança formada na terapia presencial, e que poucos artigos avaliaram a aliança como fator preditivo de resultados e de adesão. Apesar deste indicativo, este estudo discute as limitações metodológicas dos artigos e indica a necessidade de estudos que tornem mais claras as evidências sobre a formação da aliança e o o seu papel nesta modalidade alternativa de tratamento.
\end{abstract}

Palavras-chave: Videoconferência, Aliança Terapêutica, Terapia Cognitivo-Comportamental.

\section{Therapeutic Alliance in Cognitive Behavior Therapy for Videoconferencing: A Literature Review}

\begin{abstract}
Recent years have seen an increase in the number of Internet users in Brazil and in the world, while videoconferencing interventions have emerged as forms of treatment for various mental disorders. It is known that the therapeutic alliance is a key component to implement the psychotherapeutic practice; however, there is no consensus about psychotherapeutic practice and training in psychotherapy by videoconference. Therefore, this article aims to conduct a review of literature about training and the role of therapeutic alliance in Cognitive Behavior Therapy (CBT) by videoconference. For this, a search in four databases (Pubmed, Psycarticles, Lilacs and SciELO) was performed, with specific descriptors of the dictionaries of the bases. In total, 365 articles were found, but only 9 met the inclusion criteria based on the objective of this study. It was observed that most of the articles indicated that the alliance was high during the CBT sessions by videoconference and this can be compared with the alliance formed in face-to-face therapy; it was also observed that few articles evaluated the alliance as a predictor of outcomes and adherence. Despite this indicative, this study discusses the methodological limitations of the articles and indicates the need for studies that clarify the evidence about the formation of the alliance and its role in this alternative treatment modality.
\end{abstract}

Keywords: Videoconferencing, Therapeutic Alliance, Cognitive-Behavioral Therapy. 


\title{
Alianza Terapéutica en la Terapia Cognitivo-conductual por Videoconferencia: una Revisión de Literatura
}

\begin{abstract}
Resumen: En los últimos años ha habido un aumento en el número de usuarios de Internet en Brasil y en el mundo, mientras las intervenciones por videoconferencia surgieron como formas de tratamiento para diversos trastornos mentales. Se sabe que la alianza terapéutica es un componente clave para aplicar la práctica psicoterapéutica, pero no hay consenso acerca de su formación en psicoterapia por videoconferencia. Por lo tanto, este trabajo tiene como objetivo realizar una revisión de la literatura sobre la formación y el papel de la alianza terapéutica en la terapia cognitivo-conductual (TCC) para la videoconferencia. Para esto, se realizó una búsqueda en cuatro bases de datos, Pubmed, Psycarticles, Lilacs y SciELO, con descriptores específicos de los diccionarios de las bases. En total se encontraron 365 artículos, pero solo 9 cumplieron con los criterios de inclusión basados en el objetivo de este estudio. Se observó que la mayor parte de los artículos señalan que la alianza fue alta durante las sesiones de TCC por videoconferencia, pudiendo ser comparada con la alianza formada en la terapia presencial, y que pocos artículos evaluaron la alianza como un factor predictor de resultados y adherencia. A pesar de esta indicación, este estudio analiza las limitaciones metodológicas de los artículos e indica la necesidad de estudios que hagan más claras las evidencias entre la formación de la alianza y su papel en esta modalidad de tratamiento alternativo.
\end{abstract}

Palabras clave: Videoconferenci, Alianza Terapéutica, Terapia Cognitivo-Conductual.

\section{Introdução}

Nos últimos anos houve um aumento do número de usuários da internet no Brasil e no mundo (Comitê Gestor da Internet no Brasil, 2014; File, \& Ryan, 2014; Webb, Joseph, Yardley, \& Michie, 2010). Nos Estados Unidos, um levantamento realizado em 2013 pelo United States Census Bureau indicou que $83,8 \%$ das famílias norte-americanas relataram possuir computador e que $74,4 \%$ delas fazia uso da internet, sendo que, em $73,4 \%$ dos casos, a internet era de alta velocidade (File, \& Ryan, 2014). No Brasil a situação é um pouco diferente, porém o número de usuários da internet tem aumentado. Em 2008, apenas $18 \%$ dos domicílios tinham acesso à internet e, em 2013, esse número passou para $43 \%$ (Comitê Gestor da Internet no Brasil, 2014). Ao todo foram 27,2 milhões de pessoas com acesso à internet no país em 2013, número que pode ter aumentado até o ano de 2017.

A expansão do acesso à internet também afetou o meio acadêmico. Atualmente, há um crescente interesse em se investigar psicoterapias realizadas por internet para diversos transtornos mentais (Andersson et al., 2012; Knaevelsrud et al., 2014; Preschl, Maercker, \& Wagner, 2011). Tal forma de realizar psicoterapia é apontada como uma alternativa de tratamento para pessoas que não tem acesso aos serviços de saúde, inclusive populações rurais (Morland et al., 2014).

As psicoterapias por internet ou e-terapias podem ser definidas como um cuidado em saúde mental conduzido por um profissional através de e-mail, videoconferência, fóruns, chat, realidade virtual ou pela combinação destes (Manhal-Baugus, 2001). Já a psicoterapia conduzida por meio de videoconferência é uma forma de interação simultânea (síncrona) entre terapeuta e cliente. Ela é um meio de tecnologia de comunicação a distância que tem ganhado credibilidade por ser um modo conveniente e prático de tratamento psiquiátrico e psicológico (Simpson, \& Reid, 2014).

Uma das abordagens psicoterapêuticas mais utilizadas em intervenções por internet é a Terapia Cognitivo-comportamental (TCC) (Arpin-Cribbie, Irvine, \& Ritvo, 2012; Knaevelsrud, \& Maercker, 2007; Paxling et al., 2013). A TCC é um termo genérico que abrange mais de 20 abordagens de psicoterapias derivadas do modelo cognitivo e cognitivo-comportamental (Knapp, 2004). Ela tem encontrado evidências de eficácia e efetividade para o tratamento de diversos transtornos mentais (Cordioli, \& Knapp, 2008) e caracteriza-se como uma psicoterapia breve, com o foco no presente, estruturada, direcionada para resol- 
ver problemas atuais e modificar os comportamentos e pensamentos disfuncionais (Beck, 1997). Várias abordagens derivadas da TCC emergiram ao longo dos anos desde o seu surgimento (Knapp, \& Beck, 2008), mas todas assumem três pressupostos básicos comuns: o primeiro diz respeito ao papel mediacional da cognição, em que propõe que há sempre processamento cognitivo que pode influenciar respostas a eventos internos e externos; a segunda afirma que a atividade cognitiva pode ser avaliada, monitorada e medida; e a terceira defende que o comportamento almejado pode ser influenciado por meio da mudança cognitiva (Dobson, \& Scherrer, 2004).

Nas psicoterapias presenciais e por internet, evidências têm apontado que algumas variáveis do processo psicoterapêutico, tais como satisfação, adesão, atrito e, especialmente a aliança terapêutica, são fundamentais e podem ser fatores relacionados aos resultados da psicoterapia (Hedman, Andersson, Lekander, \& Ljótsson, 2015; Peuker, Habigzang, Koller, \& Araujo, 2009). Estudos mostram que a aliança terapêutica medida no início do tratamento tem sido preditora de resultado tanto em psicoterapias presenciais quanto por internet (Ormhaug, Jensen, Wentzel-Larsen, \& Shirk, 2014; Wagner, Brand, Schulz, \& Knaevelsrud, 2012).

Segundo Horvath (2000), para a abordagem cognitivo-comportamental o estabelecimento de uma aliança entre terapeuta e cliente é fundamental, pois propicia um ambiente de segurança e confiança, condições necessárias para aprender, implementar e praticar as técnicas trabalhadas em psicoterapia. A aliança terapêutica ou aliança terapêutica de trabalho é um dos componentes que constituem a relação terapêutica (Horvath, 2000). Segundo Bordin (1979), a aliança terapêutica é uma relação de colaboração mútua, marcada por concordâncias entre terapeuta e cliente e constituída por três componentes interdependentes: objetivos, tarefas e vínculo. Os objetivos dizem respeito ao consenso sobre expectativas de resultados de curto e longo prazo entre terapeuta e cliente. As tarefas podem ser definidas como acordos ou consensos entre terapeuta e cliente no que diz respeito ao que deve ser feito na terapia e como atividades diversas na terapia contribuirão para a resolução do problema do cliente. Já o vínculo está associado à ligação afetiva entre terapeuta e cliente.

Neste sentido, no campo das intervenções por internet, uma questão importante que permeia as investigações recentes relaciona-se à formação da aliança terapêutica nesta modalidade de psicoterapia e se ela poderia ser comparada com a psicoterapia presencial (Anderson et al., 2012; King, Brooner, Peirce, Kolodner, \& Kidorf, 2014). Revisões de literatura têm abordado este assunto nos últimos anos (Simpson, \& Reid, 2014; Sucala et al., 2012), mas não foram encontradas revisões que investigassem a aliança terapêutica na TCC por videoconferência.

Neste sentido, o presente estudo pretendeu realizar uma revisão da literatura sobre a formação e papel da aliança terapêutica nas TCC por videoconferência. Especificamente, buscou-se avaliar os principais indicadores bibliométricos dos estudos e, por meio da análise de conteúdo avaliar: principais características dos estudos, métodos de avaliação da aliança terapêutica, formação da aliança terapêutica na terapia por videoconferência, relação entre aliança e resultados do tratamento; e comparação da aliança entre psicoterapias por videoconferência e presenciais.

\section{Método}

A revisão foi realizada por meio da busca em quatro bases de dados: Pubmed, Psycarticles, Lilacs e SciELO. A escolha das bases de dados fundamentou-se na relação do tema com o conteúdo indexado. Para melhor definir os termos utilizados na busca foram consultados os dicionários de controle de vocabulário de cada base. Desta forma, houve divergência de termos entre as bases. A Tabela 1 mostra os termos utilizados em cada base.

Para a busca na Pubmed foi utilizado o controle de vocabulário do Medical Subject Headings (MeSH), para a Psycarticles o Thesaurus of Psychological Index Terms e, para Lilacs e SciELO, os Descritores em Ciências da Saúde (DeCS). Os termos escolhidos se referiam de forma direta ou indireta à aliança terapêutica nas intervenções por internet. O termo Therapeutic Alliance constava apenas no Thesaurus Of Psychological Index Terms, mas foi introduzido na busca em todas as bases com a finalidade de ampliar o número de artigos encontrados e melhor defini-los. Além disso, o termo Working Alliance não constava em nenhum dicionário de controle de vocabulário, mas também foi introduzido na busca das bases Pubmed, SciELO e Lilacs. Cada um dos termos utilizados foram cruzados entre si utilizando-se o operador booleano "AND" com o objetivo de restringir a pesquisa aos resumos que apresentavam ambos os termos. 
Tabela 1

Bases de dados e termos utilizados.

\begin{tabular}{lcc}
\hline \multirow{2}{*}{ Base de dados } & Aliança terapêtica & Termos \\
\cline { 2 - 3 } PUBMED & $\begin{array}{c}\text { Psychotherapeutic Processes; } \\
\text { Therapeutic Alliance; Working Alliance }\end{array}$ & $\begin{array}{c}\text { Telemedicine; Therapy, computer } \\
\text { assisted; Remote Consultation; Internet }\end{array}$ \\
PSYCARTICLES & $\begin{array}{c}\text { Psychotherapeutic Processes; } \\
\text { Therapeutic Alliance }\end{array}$ & $\begin{array}{c}\text { Computer assisted therapy; Computer } \\
\text { mediated communication; Online } \\
\text { therapy; Telemedicine; Internet }\end{array}$ \\
LILACS + SCIELO & $\begin{array}{c}\text { Psychotherapeutic Processes; } \\
\text { Therapeutic Alliance; Working Alliance }\end{array}$ & $\begin{array}{c}\text { Telemedicine; Remote Consultation; } \\
\text { Internet }\end{array}$ \\
\hline
\end{tabular}

Para a pesquisa na Pubmed, foram utilizados os termos Psychotherapeutic Processes, Therapeutic Alliance e Working Alliance para a busca dos artigos relacionados à aliança terapêutica e os termos Telemedicine, Therapy, computer assisted, Remote Consultation e Internet para definir a busca das intervenções por internet. Para a busca na base Psycarticles foram utilizados os termos Psychotherapeutic Processes e Therapeutic Alliance para definir os artigos relacionados à aliança terapêutica e os termos Computer assisted therapy, Computer mediated communication, Online therapy, Telemedicine e Internet para as intervenções por internet.

Os termos utilizados foram iguais nas bases Lilacs e SciELO. Para a busca da aliança terapêutica foram usados os termos Psychotherapeutic Processes, Therapeutic Alliance e Working Alliance e para definir os artigos sobre intervenções por internet foram usados os termos Telemedicine, Remote Consultation e Internet.

A procura nas bases foi realizada por meio da presença dos descritores selecionados em todos os campos permitidos para a busca. Foram incluídos artigos empíricos dos últimos 11 anos, de janeiro de 2004 a fevereiro de 2015, escritos nas línguas inglesa, portuguesa ou espanhola, que avaliavam a aliança terapêutica na TCC realizada por videoconferência. Foram aceitas intervenções mistas que utilizaram algumas sessões por videoconferência e outras por telefone. Foram excluídos teses, dissertações, livros, capítulos de livros, artigos teóricos ou de revisão sistemática. Também foram excluídos artigos de intervenções que não foram baseadas na TCC e inter- venções psicoterapêuticas realizadas de forma não simultânea (assíncrona).

No total, foram encontrados 296 artigos da Pubmed, 66 artigos da Psycarticles e três artigos da Lilacs, totalizando 365 artigos. Não foram encontrados artigos na base de dados SciELO. Dos 365 artigos, 133 eram duplicados entre as bases, restando um total de 232 artigos para leitura de títulos de resumos. Após esta leitura, 201 foram excluídos porque não avaliavam intervenções psicoterapêuticas por internet. Os 31 artigos restantes foram categorizados conforme a modalidade de atendimento por internet (Figura 1). Dois artigos foram classificados como "intervenções mistas", isto é, que ofereciam uma intervenção por telefone associada à psicoterapia assíncrona. Quatorze foram classificados como “terapia assíncrona”, quatro como “intervenção sem mediador (autoaplicada)" e 11 como "terapia por videoconferência (síncrona)”. Após esta etapa, foram selecionados os artigos classificados na modalidade de "terapia por videoconferência", a fim de atingir o objetivo proposto neste estudo. Os 11 estudos selecionados foram lidos na íntegra, sendo excluídos os que não eram baseados em técnicas e pressupostos da TCC, restando um total de nove artigos.

Após a leitura pelos autores, os nove artigos foram analisados com base nos seguintes indicadores bibliométricos: autoria, periódico, idioma, ano de publicação e local de realização do estudo. Visando responder aos objetivos do estudo, os autores estabeleceram categorias para proceder à análise de conteúdo dos artigos. Foram definidas as seguintes categorias: Características e métodos dos estudos - em que foram 


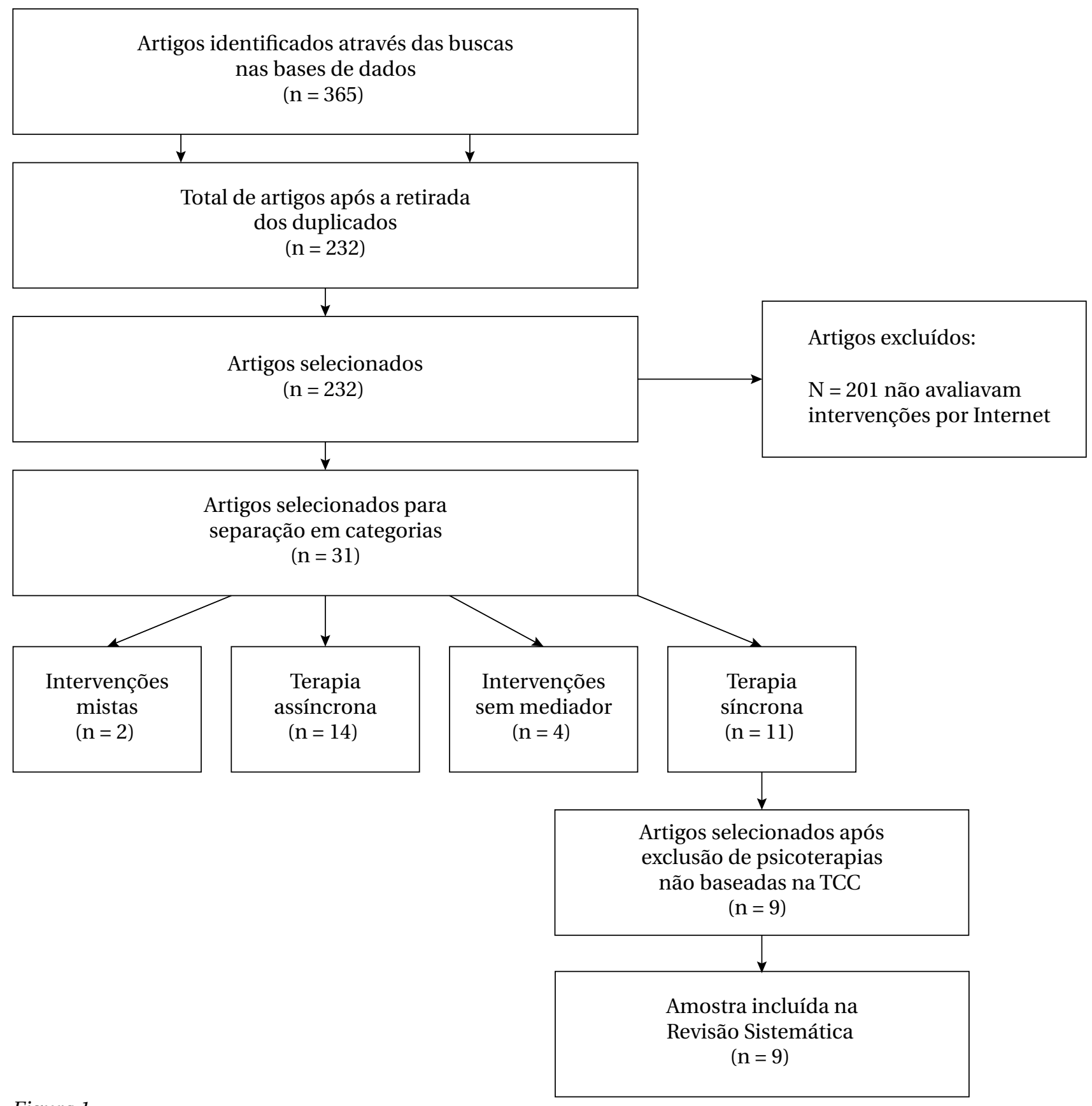

Figura 1

Diagrama dos procedimentos metodológicos utilizados para seleção dos artigos.

analisados o delineamento, metodologia, público-alvo e intervenção psicoterapêutica empregados nos estudos; Avaliação da aliança terapêutica nas TCCs por videoconferência - foram avaliadas as ferramentas utilizadas, os sujeitos avaliados e o momento do tratamento em que a avaliação da aliança terapêutica nas TCCs por videoconferência foi realizada; A formação da aliança terapêutica na TCC por videoconferência e comparação com a modalidade presencial - foi identi- ficado se houve formação da aliança e o grau em que ela ocorreu a partir dos resultados dos instrumentos. Foi avaliado também a comparação do nível da aliança entre a TCC por videoconferência e a presencial apresentadas nos resultados; Relação entre aliança terapêutica e adesão e resultados do tratamento nas TCCs por videoconferência - nesta categoria foram avaliados o papel e relação da aliança com a adesão e resultados de tratamento. 


\section{Resultados}

\section{Indicadores bibliométricos}

Todos os estudos analisados foram publicados na língua inglesa e em periódicos diferentes, a saber: Telemedicine and e-health; Cyberpsychology and behavior, The International journal of eating disorders; Journal of Consulting and Clinical Psychology; Journal of diabetes science and technology, Cyberpsychology, behavior and social networking, Journal of Clinical Psychiatry; Journal of medical internet research; e Journal of anxiety disorders. Quanto ao ano de publicação, um artigo foi publicado em 2004, um em 2008, dois em 2010, um em cada ano de 2011 e 2012, dois em 2013 e um em 2014. Quanto à autoria, todos os estudos tiveram autoria compartilhada por dois ou mais autores. Cada um dos pesquisadores Stéphane Bouchard, Leslie A. Morland, Carolyn J. Greene, Christopher B. Frueh foram autores de dois artigos desta análise. Os demais autores tiveram apenas uma publicação.

Com relação aos países de realização dos estudos, a maioria dos artigos foi produzida nos Estados Unidos $(n=5)$, seguidos do Canadá $(n=2)$, da Austrália $(n=1)$ e da Noruega $(n=1)$. O resumo das características principais dos artigos analisados e seus respectivos indicadores bibliométricos poderá ser visualizado na Tabela 2 .

\section{Características e métodos dos estudos}

Dentre os nove artigos que compuseram a amostra, seis eram ensaios clínicos randomizados (ECRs), dois eram ensaios clínicos não randomizados e um era uma série de estudos de casos. Todos os artigos realizaram análises quantitativas dos dados.

As intervenções propostas foram variadas entre os estudos. A maioria $(n=5)$ dos estudos realizou psicoterapia individual (Bouchard et al., 2004; Ertelt et al., 2011; Germain, Marchand, Bouchard, Guay, \& Drouin, 2010; Stubbings, Rees, Roberts, \& Kane, 2013; Vogel et al., 2012), mas dois artigos realizaram intervenção em grupo (Greene et al., 2010; Morland et al., 2014) e dois conduziram terapia de família (Carey, Wade, \& Wolfe, 2008; Freeman, Duke, \& Harris, 2013).

A população-alvo destes estudos também variou, sendo a mais prevalente aquela direcionada ao transtorno de estresse pós-traumático (TEPT) $(\mathrm{n}=3)$. As demais intervenções não tinham população alvo em comum. Estas intervenções focaram nos seguintes transtornos/demanda: transtorno obsessivo compulsivo (TOC), bulimia nervosa (BN) ou transtorno alimentar não especificado, transtorno do pânico com agorafobia, adultos com diagnóstico primário do Eixo-I do Manual Diagnóstico e Estatístico de Transtornos Mentais 4a Edição (DSM-IV-TR), família de jovens com Diabetes Mellitus Tipo 1 e famílias de crianças que sofreram traumatismo craniano.

Apenas um dos estudos selecionados utilizou uma intervenção mista, com algumas sessões sendo realizadas por videoconferência e outras por celular (Vogel et al., 2012), os demais utilizaram somente a videoconferência como ferramenta virtual. Quanto ao tipo de intervenção cognitivo-comportamental, cinco estudos utilizaram a TCC tradicional aplicada aos diferentes transtornos alvos das intervenções. Os quatro estudos restantes utilizaram outras abordagens de base cognitivo-comportamental, como a Terapia Familiar de Resolução de Problemas, Terapia Familiar Comportamental Sistêmica para Diabetes, Terapia de Manejo da Raiva e Terapia de Processamento Cognitivo.

\section{Avaliação da aliança terapêutica nas TCCs por videoconferência}

A aliança terapêutica foi avaliada por meio de instrumentos padronizados em diferentes momentos da psicoterapia por videoconferência. Seis estudos aplicaram o Working Alliance Inventory (WAI) (Bouchard et al., 2004; Ertelt et al., 2011; Freeman et al., 2013; Germain et al., 2010; Stubbings et al., 2013; Vogel et al., 2012), sendo que dois deles utilizaram a versão reduzida do instrumento (Stubbings et al., 2013; Vogel et al., 2012). Dois artigos utilizaram a versão abreviada da Group Therapy Alliance Scale (GTAS) (Greene et al., 2010; Morland et al., 2014) e um estudo utilizou a Agnew Relationship Measure (ARM) (Carey et al., 2008).

O WAI (Horvath, \& Greenberg, 1989) é um instrumento que utiliza uma base metateórica de relação terapêutica, e que apresenta versões para cliente, terapeuta e observador. Ele é composto por três subdimensões (objetivos, tarefa e vínculo) com 12 questões cada, avaliadas numa escala de sete itens (sempre - nunca). Sua versão reduzida contém 12 itens no total (Tracey, \& Kokotovic, 1989), selecionados a partir de análises dos três fatores que compõe a aliança terapêutica. A GTAS (Pinsof, \& Catherall, 1986) é um questionário que avalia a aliança terapêutica em grupo a partir de um modelo sistêmico de aliança. Sua versão original contém 36 itens, que seguem quatro dimensões: aliança de um membro individual do grupo com 


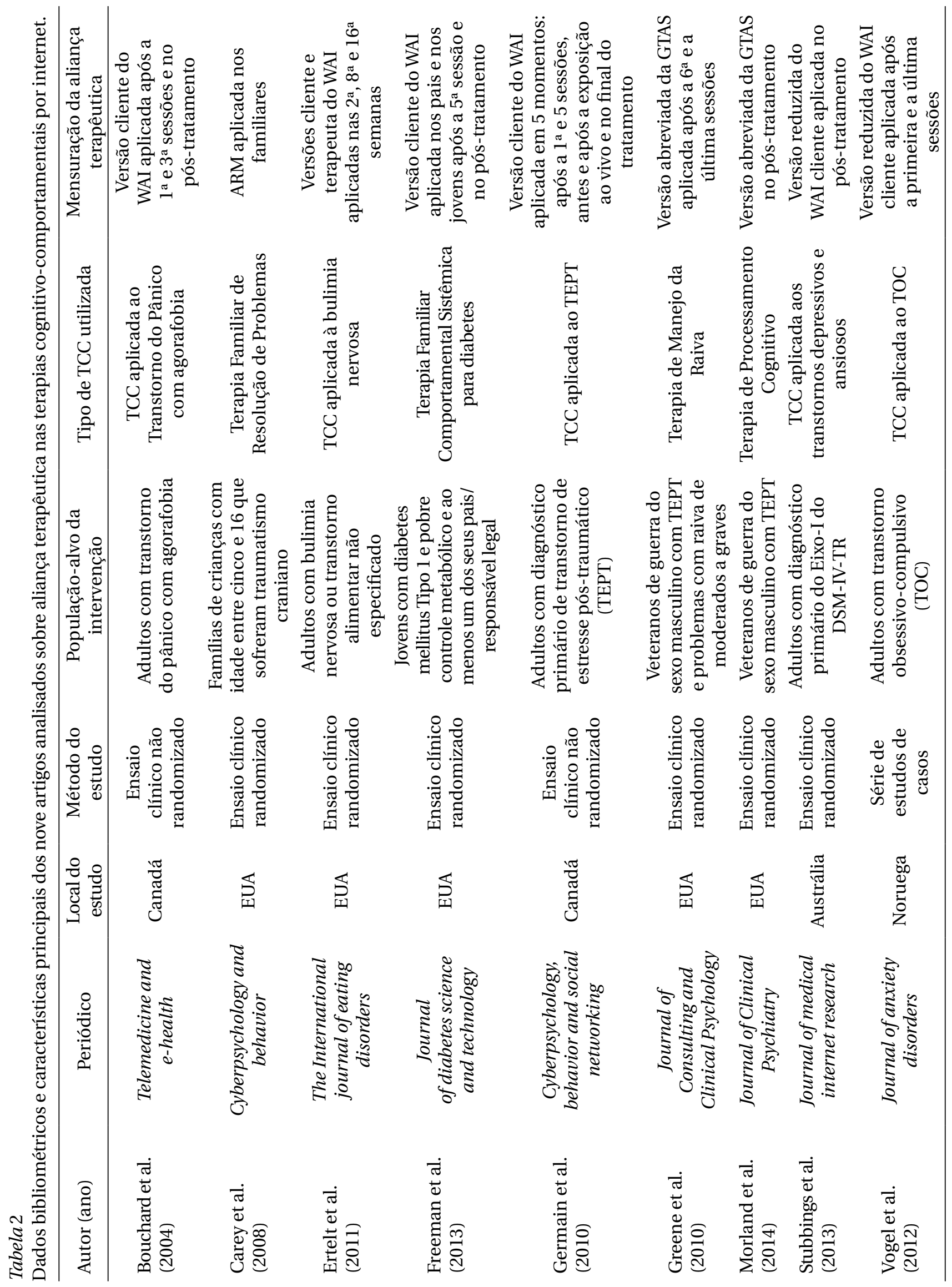


o terapeuta; aliança dos membros como um grupo com o terapeuta; aliança dos outros membros do grupo com o terapeuta; e aliança entre os membros do grupo. Os artigos que utilizaram esta escala aplicaram uma versão reduzida e adaptada da mesma para a população de interesse de cada artigo. A ARM (Agnew-Davies, Stiles, Hardy, Barkham, \& Shapiro, 1998) é um instrumento compatível com muitas abordagens de psicoterapia. Sua versão original contém 28 itens, mas o estudo que a utilizou aplicou sua versão reduzida de 12 itens (Cahill et al., 2012). Nesta versão reduzida, foram utilizados três itens de quatro subescalas: vínculo, colaboração, confiança e abertura.

A maioria dos artigos aplicou os instrumentos de avaliação da aliança apenas nos clientes $(n=8)$, somente um deles aplicou também no terapeuta e nenhum aplicou a versão do WAI observador. Em um artigo a versão do cliente foi aplicada em duas populações diferentes que participaram da intervenção, jovens com diabetes mellitus e seus familiares.

Os estudos avaliaram a aliança terapêutica em diferentes momentos do tratamento. Entre os autores que utilizaram o WAI, todos o aplicaram pelo menos uma vez após o tratamento ou na última sessão de terapia. As demais aplicações do instrumento foram bastante diversificadas (Tabela 2). Com relação ao momento de aplicação da ARM, Carey et al. (2008) não o especificaram em seu estudo. Já a GTAS foi aplicada no pós-tratamento nos dois estudos que a utilizaram, sendo que no trabalho de Greene et al. (2010) ela também foi aplicada após a sexta sessão.

\section{A formação da aliança terapêutica na TCC por videoconferência e comparação com a modalidade presencial}

Todos os estudos relataram altos níveis de aliança terapêutica nas TCCs por videoconferência. Apenas dois estudos avaliaram exclusivamente a intervenção por videoconferência e não a compararam com a psicoterapia presencial (Bouchard et al., 2004; Vogel et al., 2012).

Vogel et al. (2012) investigaram a aliança terapêutica em uma série de estudos de caso que receberam sessões de psicoterapia para TOC realizadas por videoconferência combinadas com sessões por telefone celular. Os resultados mostraram que a aliança terapêutica foi alta para todos participantes $(76 \%$ do valor máximo) e manteve-se elevada no final do tratamento.

Bouchard et al. (2004) avaliaram a aliança terapêutica na TCC por videoconferência para transtorno do pânico com agorafobia. Os resultados mostraram que as pontuações da aliança dos participantes no grupo da telepsicoterapia foram elevadas em cada avaliação (mínimo = 36; máximo = 252): 222,2 após a primeira sessão, 243,1 após a quinta sessão, e 242,4 no pós-tratamento.

Dentre os estudos que compararam a aliança terapêutica na TCC por videoconferência com a modalidade presencial, dois encontraram diferenças significativas entre estas formas de intervenção (Ertelt et al., 2011; Greene et al., 2010). Os demais manuscritos não encontraram tais diferenças, indicando que a aliança terapêutica na TCC por videoconferência foi semelhante com o tratamento presencial (Carey et al., 2008; Freeman et al., 2013; Germain et al., 2010; Morland et al., 2014; Stubbings et al., 2013).

Ertelt et al. (2011) investigaram as diferenças de fatores terapêuticos entre a TCC realizada por videoconferência ou na forma presencial para o tratamento da bulimia nervosa. Neste estudo a aliança terapêutica foi verificada tanto por meio da avaliação do cliente quanto do terapeuta. Os resultados indicaram que os clientes não identificaram diferenças significativas entre o tratamento face a face ou por videoconferência. No entanto, no que se refere às avaliações dos terapeutas, houve diferenças significativas entre os tratamentos. De modo geral, os resultados indicaram que os terapeutas fizeram classificações mais positivas das sessões na modalidade presencial.

Greene et al. (2010) avaliaram o desenvolvimento da aliança terapêutica em homens veteranos de guerra com TEPT e com problemas com raiva, que foram tratados por terapia de grupo por videoconferência ou de modo face a face. A análise das subescalas da GTAS revelou que os participantes na condição de videoconferência relataram níveis bastante variáveis e em média mais baixos de Self-Leader (percepção do participante da sua relação com o líder do grupo) quando comparados com a condição presencial. No entanto, é importante notar que a pontuação média para subescala Self-Leader na condição por videoconferência foi alta (média $=4,2$; pontuação máxima $=5$ ), sugerindo que os participantes de ambas as condições sentiam razoavelmente forte aliança em termos absolutos.

Carey et al. (2008) buscaram avaliar a efetividade da Terapia Familiar de Resolução de Problemas por Internet e identificar processos terapêuticos que possam ser influenciados pelo uso do computador, dentre eles a aliança terapêutica. Para isso, realizaram uma 
comparação entre dois grupos compostos por famílias de crianças que sofreram traumatismo craniano. Um dos grupos recebeu a Terapia Familiar de Resolução de Problemas e o outro serviu como grupo controle, recebendo informações pela internet e cuidados psicossociais presenciais. Os resultados mostraram que não houve diferenças significativas no escore geral da aliança terapêutica entre os grupos $(\mathrm{M}=72,33$; $\mathrm{DP}=$ 10,37 no grupo experimental e $\mathrm{M}=76,77 ; \mathrm{DP}=9,90$ no grupo controle; $p=0,38$ ). Os participantes do grupo da intervenção sugeriram que problemas técnicos poderiam ter atrapalhado o "andamento" da sessão, porém, isso não influenciou a aliança terapêutica.

Freeman et al. (2013) avaliaram a aliança terapêutica de jovens com diabetes mellitus do tipo 1 e seus pais com seus respectivos terapeutas, na Terapia Familiar Comportamental Sistêmica para Diabetes realizada por videoconferência ou de forma presencial. Especificamente eles investigaram se a aliança terapêutica relatada pelos jovens e seus pais difeririam entre as condições. Os resultados do teste $t$ de Student para amostras independentes não revelou diferenças significativas nos escores do WAI entre aqueles que receberam a terapia por videoconferência ou face a face. Além disso, para todas as subescalas do WAI e o escore total as respostas dos jovens foram significativamente maiores que dos pais.

Germain et al. (2010) avaliaram o impacto da TCC para TEPT, administrada por videoconferência ou presencialmente, no desenvolvimento de uma aliança terapêutica de qualidade. Os resultados apontaram que não houve diferença estatisticamente significativa na aliança terapêutica entre as duas condições, presencial e por videoconferência. A análise de efeitos simples das médias revelou uma progressão contínua em aliança terapêutica ao longo do tempo, com um ligeiro declínio no pós-tratamento, em ambas as condições. No entanto, este declínio pós-tratamento não foi significativo.

Morland et al. (2014) compararam a Terapia de Processamento Cognitivo em grupo realizada por videoconferência ou pessoalmente em uma amostra de homens veteranos de guerra com TEPT que residem em locais rurais. Os resultados indicaram altos níveis de aliança terapêutica em ambas as intervenções. Além disso, não houve diferença entre os grupos com relação à aliança terapêutica, tanto no escore total da GTAS, quanto nas suas subescalas.

Stubbings et al. (2013) investigaram a aliança terapêutica na TCC realizada por videoconferência para tratamento de Transtorno de Humor e de Ansiedade e compararam com a terapia presencial. Os resultados mostraram que não houve diferenças significativas em aliança terapêutica entre as condições, tanto nas respostas dos clientes $(\mathrm{p}=0,53)$ quanto dos terapeutas $(\mathrm{p}=0,6)$, avaliadas no pós-teste.

\section{Relação entre aliança terapêutica e adesão e resultados do tratamento nas TCCs por videoconferência}

Apenas dois estudos relacionaram a aliança terapêutica com os resultados do tratamento ou com o número de sessões concluídas na TCC por videoconferência (Freeman et al., 2013; Greene et al., 2010). Greene et al. (2010) encontraram como um dos resultados do seu estudo que alto escore na subescala da GTAS relacionada a aliança individual de um membro do grupo com o terapeuta (Self-Leader) foi associado a melhores resultados na psicoterapia. Além disso, diferenças entre as condições (presencial ou videoconferência) na subescala Self-Leader não foram suficientemente significativas para ocasionar resultados substancialmente inferiores entre os participantes na condição por videoconferência. Freeman et al. (2013) relacionaram os escores total e das subescalas do WAI, avaliados pelos participantes do estudo (pais e jovens com diabetes mellitus), com o número de sessões concluídas na psicoterapia. Os resultados mostraram que não houve associações significativas entre o número de sessões concluídas e os escores do WAI, baseados tanto nos relatos dos pais quanto dos jovens, na condição por videoconferência. Porém, o número de sessões concluídas foi positivamente associado aos escores total e da subescala "objetivo" do WAI quando relatados pelos jovens da condição presencial.

\section{Discussão}

A realização de intervenções por internet para diferentes problemas de saúde tem aumentado nos últimos anos juntamente com o crescimento do acesso à internet no Brasil e no mundo (Gomide, Martins, \& Ronzani, 2013). No Brasil, o Conselho Federal de Psicologia regulamentou, por meio da Resolução $\mathrm{n}^{\circ} 011 / 2012$, a possibilidade de realização de até 20 orientações psicológicas por internet e estabeleceu que o atendimento psicoterapêutico poderia ser realizado somente em caráter experimental. Esta Resolução vai ao encontro de uma necessidade emergente 
de se obter evidências que embasem a realização de psicoterapias e intervenções por internet do ponto de vista ético e técnico. Considerando que a aliança terapêutica é um dos alicerces da TCC e de qualquer modelo psicoterápico, faz-se necessário estudos que avaliem não somente a eficácia e efetividade destas intervenções, como também se elas são capazes de propiciar o desenvolvimento de uma aliança terapêutica entre cliente e terapeuta, como esta aliança é formada e seu papel nos resultados do tratamento. Pode-se considerar que nas intervenções simultâneas, por videoconferência, a aliança seria um alicerce tão fundamental quanto nas psicoterapias presenciais.

Atualmente podem ser encontramos na literatura um número crescente de estudos sobre intervenções por internet de modo autoaplicado, assíncrona e síncrona, para diferentes problemas (Graham et al., 2016; Zhao, Lustria, \& Hendrickse, 2017). Porém, em nossa revisão foram encontrados poucos estudos que avaliassem a aliança terapêutica nas psicoterapias assíncronas (não simultâneas) e síncronas (simultâneas), indicando a necessidade de mais estudos para elucidar sobre a aliança nesta modalidade de tratamento. Todos foram publicados em língua inglesa e realizados em países desenvolvidos, especialmente nos EUA, demonstrando a carência de estudos ou falta de interesse no tema por pesquisadores e periódicos de língua portuguesa ou espanhola. Este resultado pode estar associado à facilidade de acesso à internet e/ou ao alto investimento em pesquisa nos países desenvolvidos. Estudos que abordassem outras populações, de países em desenvolvimento, com vulnerabilidade social, ou não habituados à internet seriam de grande importância para melhor compreender a formação da aliança terapêutica. Do mesmo modo, a restrição quanto ao local do estudo interfere na generalização dos resultados.

Por outro lado, os artigos analisados foram publicados em diferentes periódicos, indicando que vários deles têm interesse no tema. Vale destacar que algumas dessas revistas publicam especificamente artigos relacionados à telemedicina e a intervenções por internet na área da saúde, como os periódicos Telemedicine and e-health; Cyberpsychology and behavior, e Cyberpsychology, behavior and social networking.

Embora tenham sido encontrados poucos estudos sobre o tema, os resultados desta revisão apontaram que todos os estudos indicaram altos níveis de aliança terapêutica nas TCCs por videoconferência. Quando a aliança foi avaliada no decorrer das sessões de psicote- rapia, ela manteve-se elevada até o final do tratamento. Contudo, cabe ressaltar que os artigos não indicaram especificamente o nível de aliança terapêutica dos que abandonaram o tratamento no decorrer das sessões, fato que pode enviesar os resultados. Os altos níveis de aliança terapêutica nas psicoterapias por videoconferência podem ser explicados, dentre outros fatores, pelo conceito de "Senso de Presença". O Senso de Presença é um conceito muito utilizado nos estudos sobre intervenções por internet e pode ser definido como "a experiência subjetiva de estar em um lugar ou em um ambiente, mesmo quando a pessoa está fisicamente localizada em outro" (Witmer, \& Singer, 1998, p. 225). No contexto da psicoterapia por videoconferência, este conceito se referiria à impressão de estar no mesmo ambiente físico do terapeuta/cliente, ao invés de estarem em ambientes físicos distintos.

Entre os estudos que compararam a psicoterapia por videoconferência com a presencial, a maioria relatou que não houve diferenças significativas entre as duas formas de intervenção, indicando que a aliança terapêutica formada na TCC por videoconferência pode ser comparada com a desenvolvida no tratamento presencial. Porém, tais resultados são inconclusivos devido ao baixo número de artigos que abordaram este tema e por haver estudos que encontraram diferença entre as intervenções (Ertelt et al., 2011; Greene et al., 2010).

$\mathrm{Na}$ amostra de artigos analisada no presente trabalho, somente dois estudos investigaram o papel ou a relação da aliança com adesão e resultados do tratamento na psicoterapia por videoconferência. Um dos estudos encontrou relação positiva entre a aliança e melhores resultados do tratamento. Porém, o outro estudo encontrou relação entre aliança e adesão somente no grupo presencial. Este resultado não converge com a literatura que aborda as psicoterapias presenciais, em que a aliança terapêutica se mostra uma variável preditiva de resultados e de adesão (Eaton et al., 1988; Liber et al., 2010). Portanto, vale salientar a importância de não fazermos inferência de que os fatores preditivos relacionados à aliança encontrados nas psicoterapias presenciais serão iguais nas psicoterapias por videoconferência. Estudos futuros poderiam avaliar melhor esta relação a fim de esclarecer se o processo é parecido.

Quanto à metodologia empregada, quase todos os estudos tiveram como desenho metodológico de pesquisa o ensaio clínico randomizado ou não, e ape- 
nas um estudo realizou um relato de uma série de estudos de caso. Os ensaios clínicos fundamentam-se numa importante ferramenta para a avaliação de intervenções para a saúde, podendo ser realizados de forma randomizada ou não (Coutinho, \& Cunha, 2005). Para o ambiente acadêmico, os ensaios clínicos randomizados são o padrão ouro de pesquisa para avaliar a eficácia de uma intervenção, o que confere aos estudos desta revisão boa qualidade no que se refere a evidências científicas. Por outro lado, apesar dos ensaios clínicos serem de grande importância para se investigar a formação da aliança terapêutica nas psicoterapias por videoconferência, compará-las com as presenciais e com resultados de tratamento, elas não são suficientes. Pesquisas qualitativas complementares poderiam esclarecer como a aliança se forma e o processo pelo qual ela se mantém ou diminui durante a psicoterapia.

A população-alvo das intervenções divergiu entre os artigos, porém, houve um predomínio de tratamentos voltados aos transtornos de ansiedade, como o TEPT, o TOC e o transtorno do pânico com agorafobia. Se por um lado são encontrados muitos estudos sobre intervenções por internet para uma variedade de populações, por outro, os resultados desta revisão mostram a necessidade de se avaliar se a aliança acontece em populações com outros transtornos psicológicos, como depressão, abuso de substâncias, fobia social, entre outros.

Quanto ao tipo de intervenção cognitivo-comportamental utilizada, a maioria utilizou a TCC tradicional aplicada aos diferentes transtornos alvos das intervenções. Porém, pôde-se observar o uso de outras psicoterapias derivadas das cognitivo-comportamentais, como a Terapia Familiar de Resolução de Problemas, a Terapia Familiar Comportamental Sistêmica para Diabetes, a Terapia de Manejo da Raiva e a Terapia de Processamento Cognitivo. As diferentes abordagens da TCC e as populações-alvo do estudo demonstram a amplitude de formas de psicoterapia que podem ser realizadas por videoconferência.

A maioria dos estudos utilizou o WAI como forma de medida da aliança terapêutica. Revisões de literatura sobre a formação da aliança em psicoterapias presenciais (McLeod, 2011) e por internet (Simpson, \& Reid, 2014) também têm demonstrado a predominância deste instrumento. OWAI é um instrumento baseado em modelo transteórico de aliança terapêutica definido por Bordin (1979) e está sendo usado como forma de mensuração da aliança em várias abordagens psicoterapêuticas (Elvins, \& Green, 2008), inclusive as que utilizam a videoconferência. Apesar disto, não foram encontrados relatos de validação deste instrumento para esta população.

Quanto ao momento de aplicação do instrumento de avaliação da aliança, todos os estudos relataram que avaliaram a aliança na última sessão ou no pós-tratamento, apenas um estudo não relatou o momento de mensuração da aliança. Os demais períodos de avaliação foram bastante distintos entre os artigos, indicando a falta de consenso em relação ao melhor momento de avaliação da aliança terapêutica no decorrer da psicoterapia. Estudos prévios indicaram que há formação da aliança nas sessões iniciais de psicoterapia (Prado, \& Meyer, 2006) e que a avaliação no início do tratamento é preditiva de resultado (Eaton, Abeles, \& Gutfreund, 1988). Desta forma, sugere-se que estudos futuros meçam a aliança também nas sessões iniciais de psicoterapia por videoconferência.

\section{Considerações Finais}

Pode-se concluir nesta revisão que a maioria dos estudos indicou que houve formação da aliança terapêutica na TCC realizada por videoconferência e que a aliança foi alta no decorrer das sessões, podendo ser comparada com a formada na psicoterapia presencial; poucos estudos avaliaram a aliança como fator preditivo de resultados e de adesão em TCC por videoconferência, mas os que tiveram este objetivo indicaram que a aliança Self-Leader estava relacionada aos resultados em TCC de grupo e que valores mais altos de aliança estavam associados à adesão apenas na psicoterapia presencial; o ensaio clínico randomizado, a análise de dados de natureza quantitativa, e a utilização do instrumento WAI foram os métodos e técnicas predominantes nos estudos.

Os resultados deste estudo sugerem que a TCC por videoconferência pode ser uma modalidade de atendimento psicoterapêutico alternativa de tratamento para pessoas que não tem acesso aos serviços de saúde (Morland et al., 2014). No entanto, ela precisa ser melhor investigada no que se refere à formação da aliança terapêutica, considerando que dentre os 31 artigos relacionados à aliança terapêutica em psicoterapias por internet, apenas nove utilizavam TCC por videoconferência. Cabe ressaltar ainda que não foram encontrados estudos sobre a aliança terapêutica e a 
realização de psicoterapia por videoconferência no Brasil. Por outro lado, há cada vez mais acesso à internet banda larga no país. Desta forma, faz-se necessário maior investimento em estudos sobre os processos e impactos da psicoterapia por internet.
Este estudo limitou-se a realizar uma revisão da literatura sobre o tema. A realização de uma metanálise no futuro pode auxiliar no entendimento dos resultados dos ensaios clínicos randomizados no que tange ao tamanho do efeito e outras variáveis estatísticas.

\section{Referências}

Agnew-Davies, R., Stiles, W. B., Hardy, G. E., Barkham, M., \& Shapiro, D. A. (1998). Alliance structure assessed by the Agnew Relationship Measure (ARM). British Journal of Clinical Psychology, 37(2), 155-172. https://doi.org/10.1111/j.2044-8260.1998.tb01291.x

Anderson, R. E., Spence, S. H., Donovan, C. L., March, S., Prosser, S., \& Kenardy, J. (2012). Working alliance in online cognitive behavior therapy for anxiety disorders in youth: comparison with clinic delivery and its role in predicting outcome. Journal of Medical Internet Research, 14(3), e88. https://doi.org/10.2196/jmir.1848

Andersson, G., Paxling, B., Wiwe, M., Vernmark, K., Felix, C. B., Lundborg, L et al. (2012). Therapeutic alliance in guided internet-delivered cognitive behavioural treatment of depression, generalized anxiety disorder and social anxiety disorder. Behaviour Research and Therapy, 50(9), 544-550. https://doi.org/10.1016/j.brat.2012.05.003

Arpin-Cribbie, C., Irvine, J., \& Ritvo, P. (2012). Web-based cognitive-behavioral therapy for perfectionism: a randomized controlled trial. Psychotherapy Research, 22(2), 194-207. https://doi.org/10.1080/10503307.2011.637242

Beck, J. (2007). Terapia cognitiva: teoria e prática. Porto Alegre, RS: Artmed.

Bouchard, S., Paquin, B., Payeur, R., Allard, M., Rivard, V., Fournier, T. et al. (2004). Delivering cognitive-behavior therapy for panic disorder with agoraphobia in videoconference. Telemedicine Journal and e-Health, 10(1), 13-25. https://doi.org/10.1089/153056204773644535

Bordin, E. S. (1979). The generalizability of the psychoanalytic concept of the working alliance. Psychotherapy: Theory, Research and Practice, 16(3), 252-260. https://doi.org/10.1037/h0085885

Cahill, J., Stiles, W. B., Barkham, M., Hardy, G. E., Stone, G., Agnew-Davies, R. et al. (2012). Two short forms of the agnew relationship measure: the ARM-5 and ARM-12. Psychotherapy Research,22(3), 241-255. https://doi.org/10.1080/10503307.2011.643253

Carey, J. C., Wade, S. L., \& Wolfe, C. R. (2008). Lessons learned: the effect of prior technology use on Web-based interventions. Cyberpsychology \& Behavior, 11(2), 188-195. https://doi.org/10.1089/cpb.2007.0025

Comitê Gestor da Internet no Brasil (2014). TIC domicílios e empresas 2013: pesquisa sobre o uso das tecnologias de informação e comunicação no Brasil. São Paulo, SP: CGI.BR.

Conselho Federal de Psicologia. (2012, 25 de junho). Resolução CFP № 011/2012. Regulamenta os serviços psicológicos realizados por meios tecnológicos de comunicação a distância, o atendimento psicoterapêutico em caráter experimental e revoga a Resolução CFP N. ${ }^{0}$ 12/2005.Diário Oficial da União.

Coutinho,E.S.F.,\&Cunha,G.M.(2005).Conceitosbásicosdeepidemiologiaeestatísticaparaaleituradeensaiosclínicos controlados. Revista Brasileira de Psiquiatria, 27(2), 146-51. https://doi.org/10.1590/S1516-44462005000200015

Cordioli, A. V., \& Knapp, P. (2008). A terapia cognitivo-comportamental no tratamento dos transtornos mentais: editorial. Revista Brasileira de Psiquiatria, 30 suppl 2, 51-53. https://doi.org/10.1590/S1516-44462008000600001

Dobson, K. S. \& Scherrer, M. C. (2004). História e futuro das terapias cognitivo-comportamentais. In P. Knapp (Org.), Terapia cognitivo-comportamental na prática psiquiátrica (pp. 42-57). Porto Alegre, RS: Artmed.

Eaton, T. T., Abeles, N., \& Gutfreund, M. J. (1988). Therapeutic alliance and outcome: Impact of treatment length and pretreatment symptomatology.Psychotherapy: Theory, Research, Practice, Training, 25(4), 536-542. https://doi.org/10.1037/h0085379

Elvins, R., \& Green, J. (2008). The conceptualization and measurement of therapeutic alliance: an empirical review. Clinical psychology review, 28(7), 1167-1187. https://doi.org/10.1016/j.cpr.2008.04.002

Ertelt, T. W., Crosby, R. D., Marino, J. M., Mitchell, J. E., Lancaster, K., \& Crow, S. J. (2011). Therapeutic factors affecting the cognitive behavioral treatment of bulimia nervosa via telemedicine versus face-to-face delivery. International Journal of Eating Disorders, 44(8), 687-691. https://doi.org/10.1002/eat.20874 
File, T., \& Ryan, C. (2014). Computer and Internet Use in the United States: 2013. American Community Survey Reports, (Nov), 1-16. Recovered from https://www.census.gov/content/dam/Census/library/publications/2014/acs/acs-28.pdf

Freeman, K. A., Duke, D. C., \& Harris, M. A. (2013). Behavioral health care for adolescents with poorly controlled diabetes via Skype: does working alliance remain intact?. Journal of Diabetes Science and Technology, 7(3), 727-735. https://doi.org/10.1177/193229681300700318

Germain, V., Marchand, A., Bouchard, S., Guay, S., \& Drouin, M. S. (2010). Assessment of the therapeutic alliance in face-to-face or videoconference treatment for posttraumatic stress disorder. Cyberpsychology, Behavior, and Social Networking, 13(1), 29-35. https://doi.org/10.1089/cyber.2009.0139

Gomide, H.P., Martins, L. F., \& Ronzani, T. M. (2013).É hora deinvestirmos em intervenções comportamentais computadorizadas no Brasil?. Psicologia em Estudo, 18(2), 303-311. https://doi.org/10.1590/S1413-73722013000200011

Graham, A.L., Carpenter, K.M., Cha, S., Cole, S., Jacobs, M.A., Raskob, M. et al. (2016). Systematic review and meta-analysis of Internet interventions for smoking cessation among adults. Substance Abuse and Rehabilitation, 7(7), 55-69. https://doi.org/10.2147/SAR.S101660

Greene, C. J., Morland, L. A., Macdonald, A., Frueh, B. C., Grubbs, K. M., \& Rosen, C. S. (2010). How does tele-mental health affect group therapy process? Secondary analysis of a noninferiority trial. Journal of Consulting and Clinical Psychology, 78(5), 746-750. https://doi.org/10.1037/a0020158

Hedman, E., Andersson, E., Lekander, M., \& Ljótsson, B. (2015). Predictors in Internet-delivered cognitive behavior therapy and behavioral stress management for severe health anxiety. Behaviour Research and Therapy, 64, 49-55. https://doi.org/10.1016/j.brat.2014.11.009

Horvath, A. O. (2000). The therapeutic relationship: from transference to alliance. Journal of Clinical Psychology, 56(2), 163-173. https://doi.org/10.1002/(SICI) 1097-4679(200002)56:2<163::AID-JCLP3>3.0.CO;2-D

Horvath, A. O., \& Greenberg, L. (1989). Development and validation of the Working Alliance Inventory. Journal of Counseling Psychology, 36(2), 222-233. https:// doi.org/10.1037/0022-0167.36.2.223

King, V. L., Brooner, R. K., Peirce, J. M., Kolodner, K., \& Kidorf, M. S. (2014). A randomized trial of Web-based videoconferencing for substance abuse counseling. Journal of Substance Abuse Treatment, 46(1), 36-42. https://doi.org/10.1016/j.jsat.2013.08.009

Knaevelsrud, C., Böttche, M., Pietrzak, R. H., Freyberger, H. J., Renneberg, B., \& Kuwert, P. (2014). Integrative testimonial therapy: an internet-based, therapist-assisted therapy for German elderly survivors of the World War II with posttraumatic stress symptoms. The Journal of Nervous and Mental Disease, 202(9), 651-658. https://doi.org/10.1097/NMD.0000000000000178

Knaevelsrud, C., \& Maercker, A. (2007). Internet-based treatment for PTSD reduces distress and facilitates the development of a strong therapeutic alliance: a randomized controlled clinical trial. BMC Psychiatry, 7(1), 13. https://doi.org/10.1186/1471-244X-7-13

Knapp, P. (2004). Terapia cognitivo-comportamental na prática psiquiátrica. Porto Alegre, RS: Artmed.

Knapp, P., \& Beck, A. T. (2008). Fundamentos, modelos conceituais, aplicações e pesquisa da terapia cognitiva. Revista Brasileira de Psiquiatria, 30 suppl 2, S54-64. https://doi.org/10.1590/S1516-44462008000600002

Liber, J. M., McLeod, B. D., Van Widenfelt, B. M., Goedhart, A. W., Leeden, A. J., Utens, E. M. et al. (2010). Examining the relation between the therapeutic alliance, treatment adherence, and outcome of cognitive behavioral therapy for children with anxiety disorders. Behavior Therapy, 41(2), 172-186. https://doi.org/10.1016/j.beth.2009.02.003

Manhal-Baugus, M. (2001). E-therapy: practical, ethical, and legal issues. CyberPsychology \& Behavior, 4(5), 551-563. https://doi.org/10.1089/109493101753235142

McLeod, B. D. (2011). Relation of the alliance with outcomes in youth psychotherapy: A meta-analysis. Clinical Psychology Review, 31(4), 603-616. https://doi.org/10.1016/j.cpr.2011.02.001

Morland, L. A., Mackintosh, M. A., Greene, C. J., Rosen, C. S., Chard, K. M., Resick, P. et al. (2014). Cognitive processing therapy for posttraumatic stress disorder delivered to rural veterans via telemental health: a randomized noninferiority clinical trial. The Journal of clinical psychiatry, 75(5), 470-476. https://doi.org/10.4088/JCP.13m08842

Ormhaug, S. M., Jensen, T. K., Wentzel-Larsen, T., \& Shirk, S. R. (2014). The therapeutic alliance in treatment of traumatized youths: Relation to outcome in a randomized clinical trial. JournalofConsultingand ClinicalPsychology, 82(1), 52-64. https://doi.org/10.1037/a0033884 
Paxling, B., Lundgren, S., Norman, A., Almlöv, J., Carlbring, P., Cuijpers, P. et al. (2013). Therapist behaviours in interne$\mathrm{t}$-delivered cognitive behaviour therapy: analyses of e-mail correspondence in the treatment of generalized anxiety disorder. Behavioural and cognitive psychotherapy,41(3), 280-289. https://doi.org/10.1017/S1352465812000240

Peuker, A. C., Habigzang, L. F., Koller, S. H., \& Araujo, L. B. (2009). Avaliação de processo e resultado em psicoterapias: uma revisão. Psicologia em Estudo, 14(3), 439-445. https://doi.org/10.1590/S1413-73722009000300004

Pinsof, W. M., \& Catherall, D. R. (1986). The integrative psychotherapy alliance: family, couple and individual therapy scales. Journal of Marital and Family Therapy, 12(2), 137-51. https://doi.org/10.1111/j.1752-0606.1986.tb01631.x

Prado, O. Z., \& Meyer, S. B. (2006). Avaliação da relação terapêutica na terapia assíncrona via internet. Psicologia em Estudo, 11(2), 247-257. httpS://doi.org/10.1590/S1413-73722006000200003

Preschl, B., Maercker, A., \& Wagner, B. (2011). The working alliance in a randomized controlled trial comparing online with face-to-face cognitive-behavioral therapy for depression. BMC Psychiatry, 11(1), 189. https://doi.org/10.1186/1471-244X-11-189

Simpson, S. G., \& Reid, C. L. (2014). Therapeutic alliance in videoconferencing psychotherapy: a review. Australian Journal of Rural Health, 22(6), 280-299. https://doi.org/10.1111/ajr.12149

Stubbings, D. R., Rees, C. S., Roberts, L. D., \& Kane, R. T. (2013). Comparing in-person to videoconference-based cognitive behavioral therapy for mood and anxiety disorders: randomized controlled trial. Journal of Medical Internet Research, 15(11), e 258. https://doi.org/10.2196/jmir.2564

Sucala, M., Schnur, J. B., Constantino, M. J., Miller, S. J., Brackman, E. H., \& Montgomery, G. H. (2012). The therapeutic relationship in e-therapy for mental health: a systematic review. Journal of Medical Internet Research, 14(4), e110. https://doi.org/10.2196/jmir.2084

Tracey, T. J., \& Kokotovic, A. M. (1989). Factor structure of the working alliance inventory. Psychological Assessment: a Journal of Consulting and Clinical Psychology, 1(3), 207.

Vogel, P. A., Launes, G., Moen, E. M., Solem, S., Hansen, B., Håland, A. T. et al. (2012). Videoconference-and cell phone-based cognitive-behavioral therapy of obsessive-compulsive disorder: a case series. Journal of Anxiety Disorders, 26(1), 158-164. https:// doi.org/10.1016/j.janxdis.2011.10.009

Wagner, B., Brand, J., Schulz, W., \& Knaevelsrud, C. (2012). Online working alliance predicts treatment outcome for posttraumatic stress symptoms in Arab War-Traumatized Patients. Depression and Anxiety, 29(7), 646-651. https://doi.org/10.1002/da.21962

Webb, T., Joseph, J., Yardley, L., \& Michie, S. (2010). Using the internet to promote health behavior change: a systematic review and meta-analysis of the impact of theoretical basis, use of behavior change techniques, and mode of delivery on efficacy. Journal of Medical Internet Research, 12(1), e4. https://doi.org/10.2196/jmir.1376

Witmer, B. G., \& Singer, M. J. (1998). Measuring presence in virtual environments: A presence questionnaire. Presence: Teleoperators and Virtual Environments, 7(3), 225-240.

Zhao, D., Lustria, M.L., \& Hendrickse, J. (2017). Systematic review of the information and communication technology features of web- and mobile-based psychoeducational interventions for depression. Patiente Education and Counseling, 100(6), 1049-1072. https://doi.org/10.1016/j.pec.2017.01.004

\section{Bianca Aparecida Ribeiro Singulane}

Psicóloga pela Universidade Federal de Juiz de Fora - UFJF e mestre pelo Programa de Pós-Graduação em Psicologia da UFJF. E-mail: bianca.singulane@gmail.com

\section{Laisa Marcorela Andreoli Sartes}

Psicóloga pela Universidade Federal de Juiz de Fora, mestre e doutora em Ciências pelo Departamento de Psicobiologia da Universidade Federal de São Paulo e professora adjunta do Departamento de Psicologia da UFJF. E-mail: laisa.sartes@gmail.com 
Endereço para envio de correspondência:

Universidade Federal de Juiz de Fora, Instituto de Ciências Humanas, Departamento de Psicologia. Rua José Lourenço Kelmer, s/n - campus, Bairro São Pedro. CEP: 36036-900.

Juiz de Fora, MG, Brasil.

Recebido 29/02/2016

Reformulação 14/03/2017

Aprovado 14/05/2017

Received $02 / 29 / 2016$

Reformulated $03 / 14 / 2017$

Approved 05/14/2017

Recebido 29/02/2016

Reformulado 14/03/2017

Aceptado 14/05/2017

Como citar: Singulne, B. A. R., \& Sartes, L. M. A. (2017). Aliança terapêutica nas terapias cognitivo-comportamentais por videoconferência: uma revisão da literatura. Psicologia: Ciência e Profissão, 37(3), 784-798.

https://doi.org/10.1590/1982-3703000832016

How to cite: Singulne, B. A. R., \& Sartes, L. M. A. (2017). Therapeutic alliance in cognitive behavior therapy for videoconferencing: a literature review. Psicologia: Ciência e Profissão, 37(3), 784-798. https://doi.org/10.1590/1982-3703000832016

Cómo citar: Singulne, B. A. R., \& Sartes, L. M. A. (2017). Alianza terapéutica en la terapia cognitivo-conductual por videoconferencia: una revisión de literatura. Psicologia: Ciência e Profissão, 37(3), 784-798.

https://doi.org/10.1590/1982-3703000832016 